BMC

Evolutionary Biology

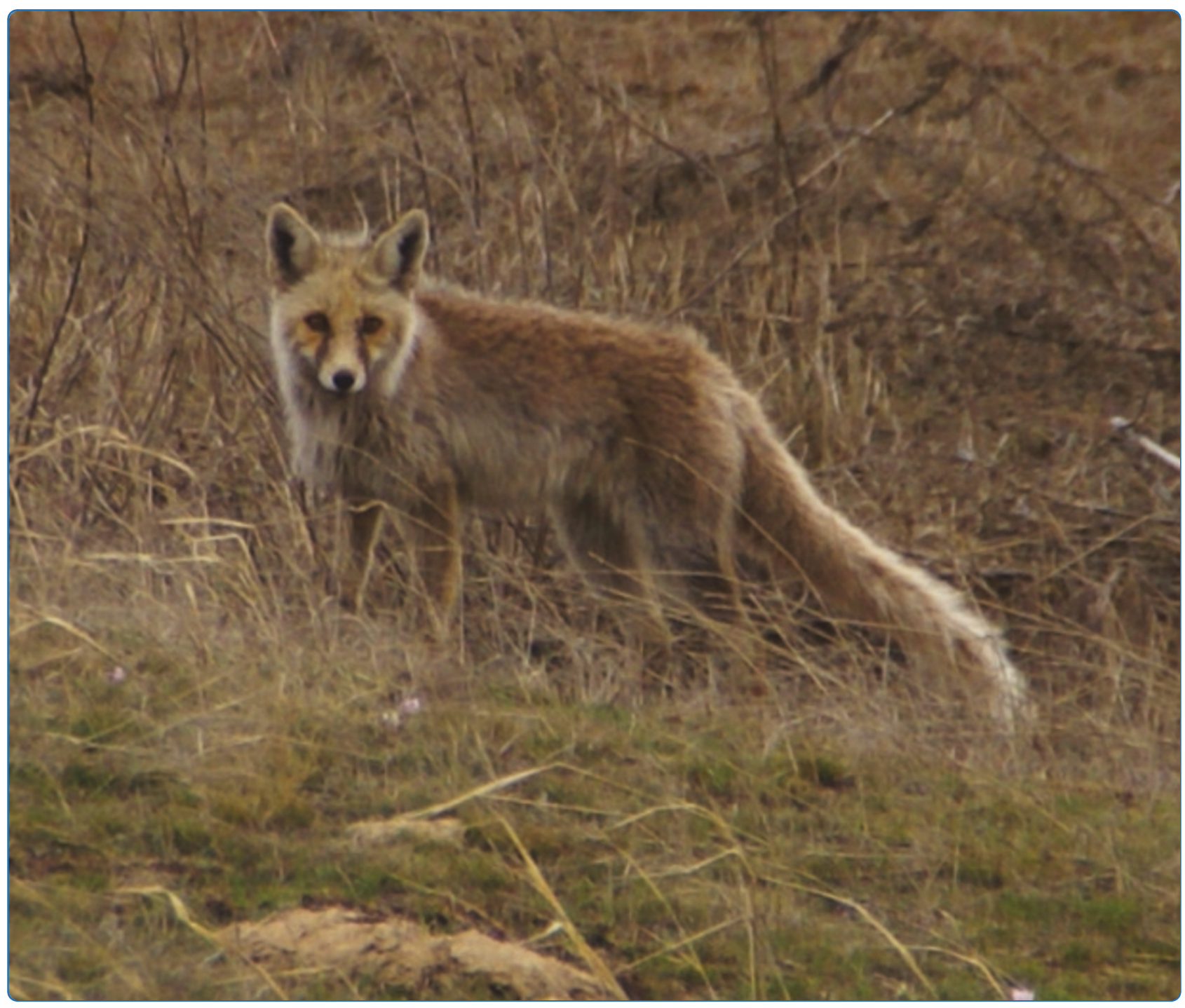

Modern and ancient red fox (Vulpes vulpes) in Europe show an unusual lack of geographical and temporal structuring, and differing responses within the carnivores to historical climatic change

Teacher et al. 


\title{
Modern and ancient red fox (Vulpes vulpes) in Europe show an unusual lack of geographical and temporal structuring, and differing responses within the carnivores to historical climatic change
}

\author{
Amber GF Teacher ${ }^{1,2^{*}}$, Jessica A Thomas ${ }^{1}$ and lan Barnes ${ }^{1}$
}

\begin{abstract}
Background: Despite phylogeographical patterns being well characterised in a large number of species, and generalised patterns emerging, the carnivores do not all appear to show consistent trends. While some species tend to fit with standard theoretical phylogeographic expectations (e.g. bears), others show little obvious modern phylogeographic structure (e.g. wolves). In this study we briefly review these studies, and present a new phylogeographical study of the red fox (Vulpes vulpes) throughout Europe, using a combination of ancient DNA sequences obtained from museum specimens, and modern sequences collated from GenBank. We used cytochrome $b(250 \mathrm{bp})$ and the mitochondrial control region (268 bp) to elucidate both current and historical phylogeographical patterning.

Results: We found evidence for slight isolation by distance in modern populations, as well as differentiation associated with time, both of which can likely be attributed to random genetic drift. Despite high sequence diversity (11.2\% cytochrome b, 16.4\% control region), no evidence for spatial structure (from Bayesian trees) is found either in modern samples or ancient samples for either gene, and Bayesian skyline plots suggested little change in the effective population size over the past 40,000 years.

Conclusions: It is probable that the high dispersal ability and adaptability of the red fox has contributed to the lack of observable differentiation, which appears to have remained consistent over tens of thousands of years. Generalised patterns of how animals are thought to have responded to historical climatic change are not necessarily valid for all species, and so understanding the differences between species will be critical for predicting how species will be affected by future climatic change.
\end{abstract}

Keywords: ancient DNA, glaciation, climate change, adaptation, carnivore

\section{Background}

Many studies have looked at the phylogeographic patterns of species, primarily using mitochondrial DNA sequence comparisons, and linked the patterns seen today to severe climatic changes in the past $[1,2]$. During the Pleistocene (approximately 2.6 million to 12,000 years ago), Europe experienced cyclical glacial and interglacial periods, with the last glacial period ending

\footnotetext{
* Correspondence: amber.teacher@helsinki.fi

'Royal Holloway University of London, Egham Hill, Egham, Surrey TW20 OEX, UK

Full list of author information is available at the end of the article
}

approximately 10,000 years ago [1]. These fluctuations in climate had profound effects on species distributions, and during glacial periods temperate species in Europe are thought to have been forced south into warmer refugial areas, primarily in Iberia, Italy and the Balkans, although other smaller cryptic refugia have also been proposed [3-6]. Following the retreat of the glaciers towards the North, species were able to recolonise the now warmer and more habitable northern regions of Europe. These patterns of range contraction and expansion have shaped the genetic diversity in modern populations through a combination of genetic drift and gene flow. 
Within Europe, genetic studies on terrestrial species typically find divergent clades which represent the refugial origins of the focal species during the glacial periods $[3,4]$. Many taxa show a deep split between Eastern and Western European clades, which likely reflects two main refugial origins in Iberia and the Italo-Balkan region [2], and generally speaking, we now have a good understanding of the routes of post-glacial expansions for various terrestrial species [1,4](Hewitt, 1999, Hewitt, 2004). Furthermore, we also have a good understanding of how these expansions occurred; theory suggests that long-distance migrants become founders of new populations $[7,8]$, replacing the previous orthodoxy which suggested that expansions were a result of substantial genetic drift $[9,10]$. A loss of genetic variability typically accompanies such range expansions, with populations that are further from a refugium tending to have lower genetic diversity [3].

However, carnivores in particular seem to show discordant patterns, with some species showing strong phylogeographical structuring, whilst others show no evidence of structuring. For example, population divergence is found in brown bears in Europe and North America $[2,11]$ and black bears in North America [12], whilst no such phylogeographical structuring is found in North American coyotes [13]. Likewise, little evidence for partitioning of haplotypes at a regional or even continental scale has been found for another carnivore, the grey wolf $[14,15]$. It seems probable that species with relatively high dispersal rates, combined with high adaptability to a range of habitats (i.e. high capabilities for migration and gene flow) may show less phylogeographical structuring. The swift fox, kit fox and arctic fox have also been shown to have little within-species phylogeographical structuring [16,17], however these species are refined to relatively small, specific habitat regions and so might not be expected to show structure within such restricted areas (see http://www. canids.org for distribution maps).

We present a phylogeographical study within Europe of a species that is widespread throughout the Northern hemisphere; the red fox (Vulpes vulpes). Previous studies on the red fox have indicated that three distinct subclades exist in North America, reflecting two recent colonisation events from North to South, and one widespread clade representing an earlier colonisation event from South to North [18]. However, an allozyme and cytochrome $b$ study of modern red fox from ten locations in Southern Europe and Israel revealed little geographical structure in this limited region [19]. Our study provides a substantially wider geographical coverage of Europe, and includes ancient DNA samples dating approximately up to 40,000 years old, allowing us to add a temporal component to the study. In this study we aimed to assess the evidence for separate clades that might be associated with different refugial origins, and whether there is any evidence for a population bottleneck and subsequent expansion (as might be related to the Last Glacial Maximum). In addition, we considered the impact of genetic drift, by looking for patterns of isolation by distance and time-associated changes in haplotypes. To approach these questions, we examined variation in two mitochondrial DNA (mtDNA) genes, cytochrome $b$ and the control region. Cytochrome $b(C y t b)$ is relatively conserved, and as such can be used to resolve deeper splits, whilst the control region harbours higher diversity and can provide higher resolution [20,21]. The use of two mtDNA gene fragments allows us to have more confidence in the results, and to help to resolve any potential ambiguities. Mitochondrial DNA is the most appropriate marker in this instance as the mutation rate is rapid enough to provide variability over the time scales in question, and recombination is very unusual, enabling straightforward interpretation of the patterns observed [21].

\section{Methods}

\section{Modern data}

Published modern red fox sequences were collated from GenBank http://www.ncbi.nlm.nih.gov for both cytochrome $b$ and the control region. Accession numbers, location information and references are listed in additional file 1, and a map is shown in Figure 1. On the occasions where multiple GenBank entries were found with an identical location and sequence, the data were collapsed into a single sequence and only one accession number is given. For incidences where a source had listed one haplotype for multiple countries, this was expanded so that there was a separate sequence for each country where this haplotype was found; multiple sites within the same country were given unique sample names.

\section{Ancient samples}

In total, 165 European red fox bone or tooth specimens dating to the late Pleistocene and early Holocene were collected from museums and colleagues (locations, dates and donors of samples successfully amplified are listed in additional file 2). Samples were drilled onto UV-sterilised aluminium foil to ensure that there was no crosscontamination, using a clean $0.8 \mathrm{~mm}$ engraving cutter. Approximately 10-40 mg of bone powder was used for DNA extraction using a standard protocol for ancient bone samples [22]. Blank extractions were performed with every batch. Extractions were performed in a separate isolated laboratory, and standard precautions were taken for working with ancient DNA [23]. Six primer pairs were designed to amplify the cytochrome $b$ and control region in six overlapping fragments for each marker, using Primer3 [24]. The cytochrome $b$ primers were as follows 


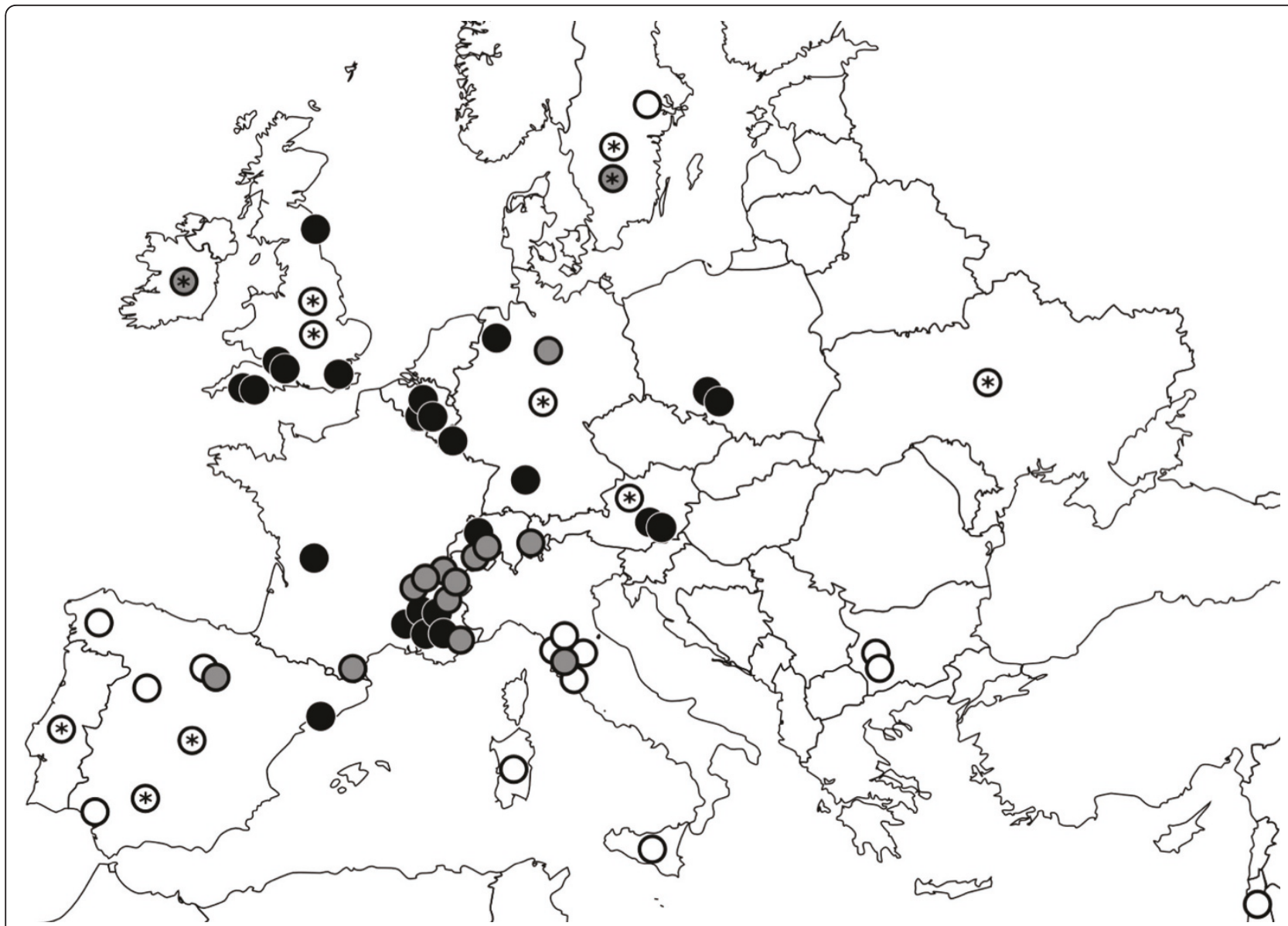

Figure $1 \mathrm{Map}$ to show sample locations. White circles indicate modern cytochrome $b$ data locations, grey circles indicate modern control region data locations, and black circles indicate ancient samples which have both cytochrome $b$ and control region data. Those with a star '*' indicate sequences for which only the country, or region within a country, is known.

(presented from 5' to 3' end): VVC1-F, GGTCCC TGCTAGGTGTATGC; VVC1-R, GTCTCGGCAGATG TGAGTGA; VVC2-F, TTGCAACAGGTCTATTTTTAG CC; VVC2-R, TAGATGCTCCGTTTGCATGT; VVC3-F, TGGCTGAATTATCCGCTACA; VVC3-R, AATTCCAATATTTCATGTTTCTATGA. The control region primers were as follows: VVD1-F, CTCCCAAAACTTGCCCTATG; VVD1-R, CTCCTGATAGAGATTATTGTAAGATT; VVD2-F, CATACTATGTTTAATCTTACAA TAATCTCT; VVD2-R, CGAGCAAGGATTGATGGTTT; VVD3-F, TCCAGTAAGGGATTTATCACCA; VVD3-R, CCTGAAGTAAGAACCAGATG.

Primers were initially tested for efficacy using modern samples (donated by Dr. Peter Wandeler, Universität Zürich-Irchel and Dr. Frank Zachos, ChristianAlbrechts-Universität Zu Kiel). Samples were amplified using PCR with the following reaction mixture at a total volume of $25 \mu \mathrm{l}: 15.1 \mu \mathrm{l}$ sterile double-distilled water, $2.5 \mu \mathrm{l}$ BSA (1\%), $0.4 \mu \mathrm{l}$ each primer at $10 \mathrm{pmol}$ (MWG, Germany), $0.25 \mu \mathrm{l}$ dNTP mix (Invitrogen, UK), $0.3 \mu \mathrm{l}$
Taq Platinum (Invitrogen, UK), $2.5 \mu \mathrm{l}$ PCR buffer (supplied with Taq), $1 \mu \mathrm{l} \mathrm{MgSO}_{4}$ (supplied with Taq), $4 \mu \mathrm{l}$ DNA. The samples were denatured at $95^{\circ} \mathrm{C}$ for $10 \mathrm{~min}-$ utes followed by 40 cycles of $94^{\circ} \mathrm{C}$ for $30 \mathrm{sec}, 50^{\circ} \mathrm{C}$ for $30 \mathrm{sec}$, and $72^{\circ} \mathrm{C}$ for $30 \mathrm{sec}$, followed by a final elongation step of $72^{\circ} \mathrm{C}$ for $7 \mathrm{~min}$ to complete fragment extension. Negative controls were run using blank extraction samples for each PCR run. Purification and sequencing was outsourced to Beckman-Coulter Genomics (Essex, UK). Six overlapping sequences were obtained for each gene, and all sequences were inspected manually to ensure that they were correctly scored.

\section{Phylogenetic analyses}

Sequences were aligned using Sequencher v.4.8 (Gene Codes Corporation, Michigan, USA) and the resulting contigs consisted of 250 base pairs cytochrome $b$ and 268 base pairs control region for the 35 ancient samples which successfully amplified at all 12 fragments. Modern sequence data was trimmed to cover the same regions. 
All ancient samples had sequence for both gene fragments, but because the modern sequences were obtained opportunistically from GenBank, we were not able to obtain sequence data for both markers for the majority of the modern samples.

We used jModeltest v.0.1.1 [25,26], and the Bayesian Information Criterion (BIC), to select the best-fit model of evolution for each gene fragment individually. A Bayesian consensus tree of the sequences was constructed using MrBayes v.3.1.2. [27]. Cytochrome $b$ and control region sequences were concatenated for each individual, and for modern individuals where both gene sequences were not available the sequences were simply coded as missing data. The data was run as a partitioned dataset, with the evolutionary model for each gene (as determined by jModelTest) coded in, and allowing for each partition to evolve under a different rate. Only those models that did not include a proportion of invariant sites were considered, owing to the intra-specific nature of the data. Sampling was set to every 100 generations for $3 \times 10^{6}$ generations with four chains (average standard deviation of split frequencies $<0.02$ ). We coded the chosen model of nucleotide substitution, and excluded the first $25 \%$ of samples as burn-in. The arctic fox (Alopex lagopus) was used as an outgroup (Accession numbers: Cytochrome $b$ AY598511, control region DQ500882). To see if they provided any additional information, maximum likelihood trees were also produced using MEGA version v5.0 [28], using 500 bootstraps and the HKY model of evolution for the following datasets: (i) Cyt $b$ modern samples, (ii) Cyt $b$ modern+ancient samples combined, (iii) control region modern samples, (iv) control region modern +ancient samples combined, and (v) Cyt $b$ and control region combined for ancient samples.

Sequences were then collapsed into haplotypes using TCS v.1.21 [29], converted to Arlequin format [30] using FaBox v.1.35 online [31], and the resulting files were used to obtain network distance connections using Arlequin v.2.000 [30]. Networks were then drawn separately for each gene fragment (as it is not possible to allow for a partitioned dataset for such analyses) using HapStar [32].

\section{Isolation by distance}

In order to check for a geographical signal within our dataset, we correlated genetic distance to geographical distance. Pairwise nucleotide distance matrices were produced for seven datasets using MEGA v.5 [28]: (i) Cyt $b$ modern samples, (ii) Cyt $b$ ancient samples, (iii) $C y t b$ modern+ancient samples combined, (iv) control region modern samples, (v) control region ancient samples, (vi) control region modern+ancient samples combined, and (vii) Cyt $b$ and control region combined for ancient samples. Approximate co-ordinates were identified for each site (see additional files 1 and 2) using Google Earth http://www.google.com/earth/index. html, and geographical distance matrices were created for each dataset using the Geographical Distance Matrix Generator [33]. Mantel tests were performed for each dataset using the Isolde function in GenePop on the Web [34,35], with 10,000 permutations.

\section{Temporal analyses}

Past population dynamics can be reconstructed by estimating demographic parameters using the coalescent approach implemented in BEAST [36]. Ancient DNA can provide both temporal and phylogenetic information through the use of tip-dated sequences. In order to determine whether there was sufficient temporal signal in the data to estimate substitution rates accurately, we first conducted a date randomisation test following Firth et al. [37]. This test involves generating 20 datasets in which the tip-dates of each sequence are randomly shuffled. The estimates of substitution rate from these 'randomised' datasets can then be compared to the rates estimated for the 'real' dataset. The presence of sufficient temporal signal is assessed by determining whether the mean rate estimates of the real dataset fall outside the $95 \%$ highest posterior density (HPD) intervals for the rate estimates of the randomised datasets. Ancient sample ages for tip-dating were inferred through stratigraphic correlation (layer dating); any samples without dates, or with approximate or infinite ages were not included in the BEAST analysis (see additional file 2). The arctic fox outgroup A. lagopus was also excluded. For the BEAST analysis, models of sequence evolution for each partition were determined in jModelTest $[25,26]$, with the nucleotide frequencies and substitution rate parameters estimated from the data. A strict clock was assumed (linked across partitions), with a constant size coalescent tree prior.

The dynamics of population size through time can be estimated using the Bayesian skyline plot in BEAST [36]. This analysis was run for 50 million generations, with parameters (described above) and genealogies sampled every 10,000 iterations and the first 20\% discarded as burnin. As a number of sequences were obtained from layer-dated samples, with their ages given as ranges rather than as point estimates; uniform priors were used to model the uncertainty in these ages, rather than point-estimates (see additional file 2).

\section{Results}

We successfully sequenced all six ancient DNA fragments for 35 samples out of the 165 collected, though some small sequence gaps were present (Figure 1, additional file 2, GenBank accession numbers JN232446JN232515; this success rate of $21 \%$ is similar to comparable ancient DNA studies [e.g. [38,39]]. The successful 
samples represent 24 localities and 9 countries. We also obtained 38 modern cytochrome $b$ sequences from GenBank, from 22 localities in 12 countries, and 39 control region sequences from 15 localities in 7 countries. Sequence diversity was high within the datasets; 26 haplotypes were identified in the cytochrome $b$ sequences (modern + ancient), and 47 haplotypes in the control region sequences. We identified 28 (11.2\% of bases) and 44 (16.4\% of bases) polymorphic sites in the cytochrome $b$ and control region fragments respectively.

\section{Phylogenetic analyses}

The jModelTest analyses determined that the least complex model of evolution with the best fit to the cytochrome $b$ data was the HKY model $(\mathrm{Ti} / \mathrm{Tv}=6.4802$, unequal base frequencies freq $\mathrm{A}=0.289$, freq $\mathrm{C}=0.231$, freq $=0.156$, freq $\mathrm{T}=0.324)$, and for the control region was the TIM $2+\mathrm{G}$ model (unequal base frequencies freqA $=0.295$, freqC $=$ 0.241 , freqG $=0.137$, freqT $=0.328$, unequal substitution rates $\mathrm{r}_{\mathrm{AC}}=0.244, \mathrm{r}_{\mathrm{AG}}=5.916, \mathrm{r}_{\mathrm{AT}}=0.244, \mathrm{r}_{\mathrm{CG}}=1.000$, $\mathrm{r}_{\mathrm{CT}}=2.133, \mathrm{r}_{\mathrm{GT}}=1.000$, scaled to $\mathrm{r}_{\mathrm{GT}}$, and gamma shape distribution $=0.130$ ).

The Bayesian tree (Figure 2) showed no obvious geographically or temporally based clades, despite reasonable sequence diversity (see above). Although some smaller clades can be seen, the posterior probabilities do not show particularly high support, all but one (Luxembourg) of the locations found in the smaller clades are also found in the larger main clade (England, France, Germany, Spain, Austria, Poland and Switzerland), and it is of note that the most basal sample is not an ancient sample, but a modern Austrian sample. The maximum likelihood trees were less informative than the Bayesian tree, with very low bootstrap support values (typically $<60$ ) and consequently have not been included. Haplotype networks for cytochrome $b$ and the control region (Additional files 3 and 4) also show no indication of haplotype clustering, and both networks show a star-like construction, which is consistent with gradual population expansion. Furthermore, there appears to be no clustering by sample age; ancient samples do not seem to cluster together, nor do they appear only at the centre of the networks (as might indicate an expansion event from the ancient samples), nor do they appear only at the edges of the networks (as could indicate a bottleneck). Specifically, it is worth noting that the oldest samples (Austria $30 \mathrm{ka}$, Austria 35-40 ka and England 59$28 \mathrm{ka})$ do not cluster together.

\section{Isolation by distance}

We detected a slight but statistically significant signal of positive isolation by distance in one dataset: (iv) control region modern samples $\left(\mathrm{R}^{2}=0.048, p\right.$ (one-tailed $)=$ 0.026). No evidence for isolation by distance was detected for the remaining datasets: (i) Cyt $b$ modern samples, (ii) Cyt $b$ ancient samples, (iii) Cyt $b$ modern +ancient samples combined, (v) control region ancient samples, (vi) control region modern+ancient samples combined, and (vii) Cyt $b$ and control region combined for ancient samples.

\section{Temporal analyses}

The models of sequence evolution for the BEAST analysis were determined by jModelTest as the HKY model for the cytochrome $b$ data and the TIM1+G model for the control region (this differs slightly from the cytochrome $b$ model used for the phylogeny, as a reduced dataset comprising of only dated samples was used for the BEAST analysis; see additional file 2). The date randomisation test indicated there was sufficient temporal signal in the data to estimate substitution rates, with the mean rate estimates for the real datasets falling outside the 95\% HPD intervals of the date-randomised datasets (see additional file 5). The mean substitution rate was estimated as $2.987 \times \mathrm{E}-7$ substitutions/site/year. The Bayesian skyline plot (Figure 3) indicates a marginal increase in population size over the last 40,000 years, compatible with the hypothesis that there was no substantial change in red fox population size during the last glacial maximum and into the Holocene.

\section{Discussion}

We detected signals of isolation by distance (in modern control region samples), as well as differentiation associated with time, but we did not detect any evidence for a historical bottleneck or for phylogeographical structure within Europe. Furthermore, this lack of structure appears to have been consistent over tens of thousands of years, though it is also possible that structure existed historically but that we were unable to detect it. Our results imply that low levels of random genetic drift have caused slight changes over space and time, but that fox populations in Europe were most likely not forced into glacial refugia, instead managing to survive the glaciations as a single large interbreeding population.

\section{Isolation by distance and time}

Evidence for isolation by distance was only detected in the modern control region sequences; however it is probable that the modern cytochrome $b$ data simply did not have the resolution to detect this pattern. No isolation by distance was detected in any of the ancient datasets tested (or the combined modern and ancient datasets for either gene), most likely because while the modern dataset represents a snapshot in time, the ancient samples come from a very wide time range, and so patterns of isolation by distance may be obscured. The degree of isolation by distance detected most likely represents the effects of random genetic drift. 


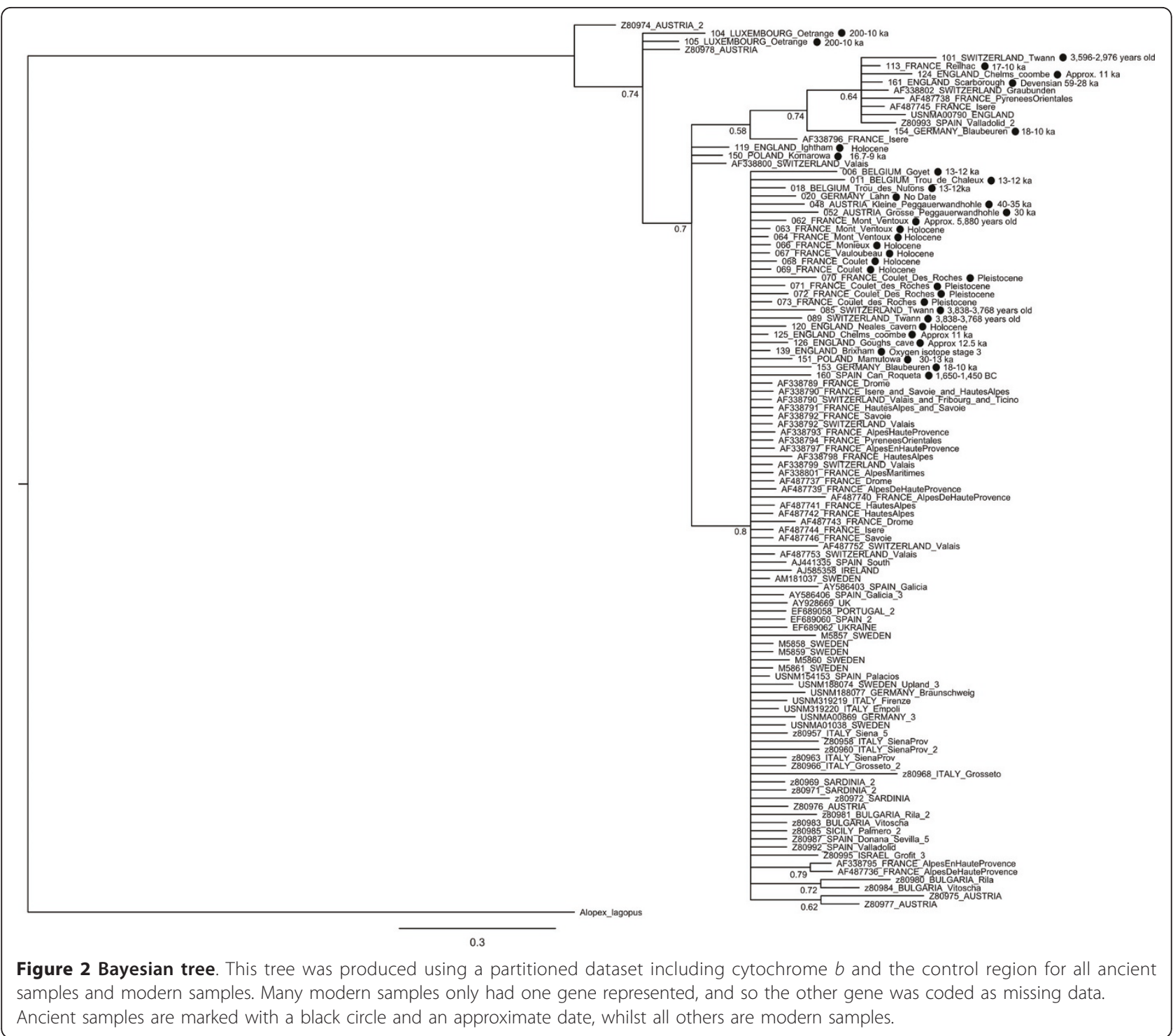

Date randomisation tests revealed differentiation associated with time, i.e. there is a detectable correlation between genetic distance and temporal distance.

Several haplotypes are shared between modern and ancient samples (despite several tens of thousands of years separating some of the samples), and the ancient samples do not form a separate or basal clade, providing supporting evidence that changes over time have not been severe. Again, these changes over time can probably be attributed to random genetic drift.

\section{Lack of phylogenetic structure or evidence for a bottleneck}

Phylogenetic studies frequently detect separate lineages in Europe which reflect population bottlenecks, isolation in glacial refugia, and subsequent recolonisation.
However, we found no evidence for separate lineages within our data, and our Bayesian skyline plots indicate a relatively constant population size. Furthermore, there is no evidence of a severe loss of unique haplotypes dating to before the Last Glacial Maximum (LGM, circa 23,300 to 27,800 years ago [40]) as might be expected from a bottleneck (Figure 3). These results combined suggest that the red fox was present in Europe throughout the glaciation, rather than being forced into glacial refugia with re-emergence and population expansion during the warmer periods. However, it is also possible that historical population structure may have existed but is undetected in our study, perhaps due to a relatively small sample size from each time period. Undetected historical phylogeographic structure could have since been reduced by gene flow, leading to a lack of structure in modern samples. 


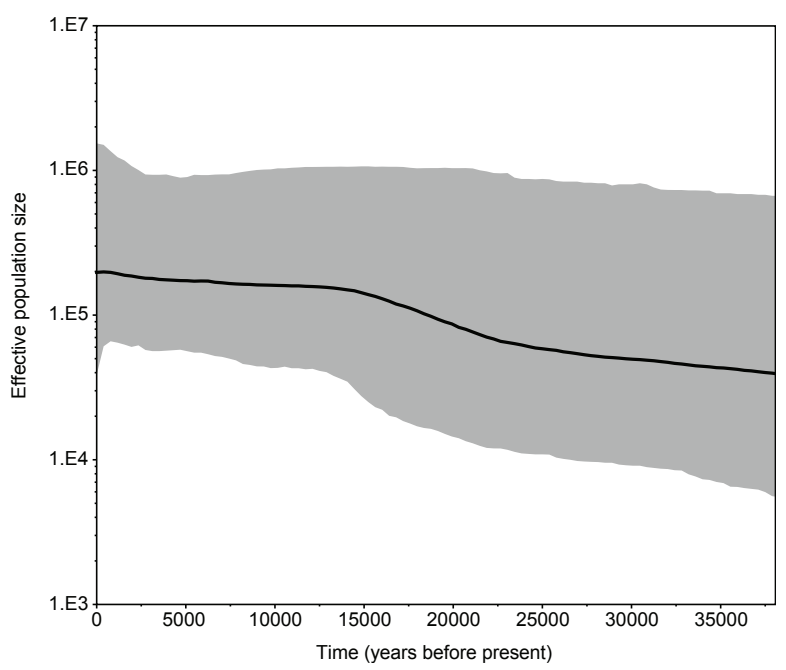

Figure 3 Bayesian skyline plot showing red fox effective population size through time. Median estimates are shown by the solid black line, and 95\% HPD intervals are indicated by the surrounding grey area.

Our findings provide strong support for those of previous smaller scale studies, which found little genetic structuring in modern red foxes in the South of Europe and Israel [19], or in France [41]. Our findings do however appear to be contradictory to a previous study in the red fox which found some evidence for phylogeographic structuring in North America [18], however it is probable that this difference reflects the very different histories of the two regions. American red foxes are thought to have been isolated for a long time (during the Wisconsin glaciation, between 110,000 and 10,000 $\mathrm{BP})$ in two disjunct refugia [42,43], However, there is no evidence for such a prolonged glaciation and subsequent isolation of populations in Europe; as such, the results of our study and that of Aubry et al. [18] are not necessarily contradictory to each other. It is probable that two characteristics of the red fox may have contributed to the lack of structuring found in our study; a high dispersal rate, and high adaptability to different habitats.

\section{Dispersal and adaptability}

Dispersal ability is expected to have a strong effect on genetic differentiation, as high dispersal can mean high gene flow and thus lower levels of differentiation. The red fox has a highly flexible social system, with both dispersing and non-dispersing males and females [44]. Those that do disperse are known to do so over large distances, for example a mark-recapture study showed that a single fox, tagged as a pup, had dispersed $302 \mathrm{~km}$ in North Dakota [45]. Mean dispersal distances of tagged pups were quoted within the same study as $35 \mathrm{~km}$ for males and $25 \mathrm{~km}$ for females, with the majority of recoveries of tagged individuals occurring by 1 year. Another species that shows little phylogeographic structure, the grey wolf, also has a very high dispersal rate, with movements of over 1,000 km recorded for an individual [14], and coyotes also show low structuring and high dispersal (50-100 km) [13,46,47]. However, dispersal rates alone are not enough to explain why some carnivores show phylogeographic structure whilst others do not. The degree of specialisation versus adaptability is also likely to play a key role. The red fox is highly adaptable, as exemplified by a range that encompasses almost the entire Northern hemisphere, including regions above the arctic circle, and South as far as Yemen and Northern India [48]. Sub-fossil assemblages indicate that between 38-25 BC (i.e. before the LGM), the distribution of the red fox is likely to have covered central Europe, and at least as far north as Southern England [49]. A radiocarbon date from the UK of $28,641 \pm 468$ cal BP [50] confirms that this species was present in Northern Europe up to a very short time before the LGM. Following the LGM, and the retreat of the glaciers, the red fox is thought to have quickly returned to central Europe, and fossils have been found within assemblages that date to 15,500 BP in northern Germany [51], and from 16,000$15,500 \mathrm{BP}$ in south-eastern Germany [52]. When this information is combined, it seems that the red fox was likely only forced into the warmer South for a short period during the LGM. Iberia, Italy, Southern France, the Balkans, the Carpathians and the Crimean peninsula have all been suggested as possible glacial refugia for the red fox, based on the sub-fossil record [reviewed in $[49,53]]$. However, there are records of this species being present in Spain, Portugal, France, Italy, Austria, Hungary, Czech Republic, Slovakia and Slovenia during the LGM, indicating a wide and continuous range during this period [53]. This information from the fossil record, and circumstantial evidence that this species highly adaptable and able to survive in sub-arctic conditions today, both support our genetic findings in indicating that the red fox may not have been strictly restricted to refugia during the European glaciations.

Differences in dispersal ability and adaptability can cause very different outcomes, as respectively exemplified in the following two species studies, brown bears and artic fox. In a study on brown bears (Ursus arctos), Valdiosera et al. [38] showed that although bears in modern Europe do show differentiation into major lineages, this pattern breaks down in ancient samples. The authors concluded that this highly adaptable species, with its very flexible diet, was able to survive across a large area in Southern Europe during the glaciations, as we are suggesting was also the case for the red fox. However, in contrast our findings for the red fox, brown bear lineage separations occurred later, most likely due to low 
dispersal distances and decreasing population sizes. A rather different pattern can be found within the Arctic fox however, which is a relatively specialist species but with good dispersal abilities. A recent phylogeographic study on the arctic fox (Alopex lagopus) examined the haplotypes that were present in Europe during the Pleistocene and compared these with the present-day haplotypes. This study indicated that arctic fox populations from mid-latitude Europe became extinct as the climate warmed at the end of the last ice-age, and that this species did not track its arctic habitat as it shifted North to Scandinavia; the present-day Scandinavian arctic foxes are thought to originate from a later expansion event from Siberia [39]. This is strongly in contrast to our findings from red fox, which indicate little change in haplotypes present despite substantial climatic change.

\section{Conclusions}

To the best of our knowledge, this is the first time that a study has found such a spatially and temporally wide-scale lack of phylogeographic structure in a terrestrial species within Europe. Our study adds to a collection of phylogeographical studies on carnivores, which when looked at together indicate that there may be a difference in response to historical climatic change between species with differing traits such as dispersal rates and adaptability. It seems likely that the high dispersal abilities, and adaptability to a wide habitat range, has led to the lack of observable differentiation in the red fox, and it is possible that such traits mean that this species may respond well to future climatic changes. However this also raises our awareness that quite similar species can respond very differently.

A comprehensive study into the life-history traits of carnivores, including their niche breadth, dispersal abilities, home range, body size, and responses to historical climatic change would perhaps be enlightening, allowing a deeper understanding of the traits that determine how a species is likely to be affected by changes in habitat availability.

Realising that generalised patterns are unlikely to be applicable to all species, and understanding the differences between how species responded to historical climatic changes may help us to create better predictions of how animal species will be affected by climate changes in the future.

\section{Additional material}

Additional file 1: Modern sequence information. GenBank accession numbers, location information, and number of sequences used in modern phylogeny.

Additional file 2: Ancient sample information. Ancient samples, locations, museum codes, sample donors, and dating information, including the approximations of the dates used for the BEAST analysis.
Additional file 3: Cytochrome $\boldsymbol{b}$ haplotype network. White circles represent modern sequences, black circles represent ancient sequences, and split circles represent haplotypes present both in ancient and modern samples. Each haplotype has a unique number, and the country of origin is marked nearby, together with the number of sequences represented in brackets. Details for the central haplotype are shown to the side. For ancient samples, where available the approximate date of the sample is also given in brackets; those marked with a star ${ }^{\prime * \prime}$ are dated by dendrochronology, the one sample marked with two stars ${ }^{\prime * * \prime}$ is radiocarbon dated, and all others are contextual dates.

Additional file 4: Control region haplotype network. White circles represent modern sequences, black circles represent ancient sequences, and split circles represent haplotypes present both in ancient and modern samples. Each haplotype has a unique number, and the country of origin is marked nearby, together with the number of sequences represented in brackets. For ancient samples, where available the approximate date of the sample is also given in brackets; those marked with a star ${ }^{\prime * \prime}$ are dated by dendrochronology, the one sample marked with two stars ${ }^{\prime * * \prime}$ is radiocarbon dated, and all others are contextual dates.

Additional file 5: Marginal probability distributions for real and randomised datasets. Marginal probability distributions for real and randomised datasets, with the mean substitution rate of 2.987 substitutions per site per year indicated for the real dataset. Real datasets are indicated in black, randomised are in colour.

\section{Acknowledgements and Funding}

Many thanks to Simon Ho for helpful suggestions that have undoubtedly improved this manuscript, and to two anonymous referees who have also helped us to improve this work. Thanks also to the museum curators and researchers who very generously donated specimens for this study: Peter Wandeler, Frank Zachos, Evelyne Cregut, Oliver Hampe, Florian Fladerer, Marc Nussbaumer, Christine Argot, Pascal Tassy, Andy Currant, Adam Nadachowski, Grzergorz Lipecki, Henriette Obermaier, Jordi Lorenzo, Ursula Gohlich, Evangelia Tsoukala, Raffaele Sardella, and Dominique Bonjean. This research contributes towards the CLIMIGRATE project, and was funded by NERC grant NE/G00269X/1 through the European Union FP7 ERA-NET program, BiodivERsA.

\section{Author details}

${ }^{1}$ Royal Holloway University of London, Egham Hill, Egham, Surrey TW20 OEX, UK. ${ }^{2}$ Department of Biosciences, P.O. Box 56 (Viikinkaari 9), Fl-00014 University of Helsinki, Finland.

\section{Authors' contributions}

AGFT, JAT and IB conceived the study, AGFT collected the samples and performed the molecular work, AGFT and JAT analysed the data, AGFT wrote the manuscript, AGFT, JAT and IB contributed and finalised subsequent drafts.

Received: 18 March 2011 Accepted: 20 July 2011

Published: 20 July 2011

\section{References}

1. Hewitt GM: Genetic consequences of climatic oscillations in the Quaternary. Philos T Roy Soc B 2004, 359:183-195.

2. Taberlet P, Fumagalli L, Wust-Saucy AG, Cosson JF: Comparative phylogeography and postglacial colonization routes in Europe. $\mathrm{Mol}$ Ecol 1998, 7:453-464.

3. Hewitt G: The genetic legacy of the Quaternary ice ages. Nature 2000 405:907-913.

4. Hewitt GM: Post-glacial re-colonization of European biota. Biol J Linn SoC 1999, 68:87-112.

5. Stewart JR, Lister AM: Cryptic northern refugia and the origin of modern biota. TREE 2001, 16:608-613.

6. Stewart JR, Lister AM, Barnes I, Dalen L: Refugia revisited: individualistic responses of species in space and time. In P Roy Soc Lond B. Volume 277. University of Geneva; 2010:661-671, 2000. 
7. Ibrahim KM, Nichols RA, Hewitt GM: Spatial patterns of genetic variation generated by different forms of dispersal during range expansion. Heredity 1996, 77:282-291.

8. Nichols RA, Hewitt GM: The genetic consequences of long distance dispersal during colonization. Heredity 1994, 72:312-317.

9. Skellam TG: Random dispersal in theoretical populations. Biometrika 1951, 38:196-218.

10. Van den Bosch F, Zadocks JC, Metz JA: Focus expansion in plant disease. II: Realistic parameter-sparse models. Phytopathology 1988, 78:59-64.

11. Waits LP, Tablot SL, Ward RH, Shields GF: Mitochondrial DNA phylogeography of the North American brown bear and implications for conservation. Conserv Biol 1998, 12:408-417.

12. Wooding S, Ward R: Phylogeography and Pleistocene evolution in the North American black bear. Mol Biol Evol 1997, 14:1096-1105.

13. Roy MS, Geffen E, Smith D, Ostrander EA, Wayne RK: Patterns of differentiation and hybridization in North American wolflike canids revealed by analysis of microsatellite loci. Mol Biol Evol 1994, 11:553-570

14. Vila C, Amorim IR, Leonard JA, Posada D, Castrovieko J, Petrucci-Fonseca F, Crandall KA, Ellegren H, Wayne RK: Mitochondrial DNA phylogeography and population history of the grey wolf Canis lupus. Mol Ecol 1999, 8:2089-2103.

15. Pilot M, Branicki W, Jędrzejewski W, Goszczyński J, Jędrzejewska B, Dykyy I, Shkvyrya M, Tsingarska E: Phylogeographic history of grey wolves in Europe. BMC Evol Biol 2010, 10:104.

16. Dalén L, Fuglei E, Hersteinsson P, Kapel CMO, Roth JD, Samelius G, Tannerfeldt $M$, Angerbjörn A: Population history and genetic structure of a circumpolar species: the arctic fox. Biol I Linn Soc 2005, 84:79-89.

17. Mercure A, Ralls K, Koepfli KP, Wayne RK: Genetic subdivisions among small canids: Mitochondrial DNA differentiation of Swift, Kit, and Arctic foxes. Evolution 1993, 47:1313-1328.

18. Aubry KB, Statham MJ, Sacks BN, Perrine JD, Wisely SM: Phylogeography of the North American red fox: vicariance in Pleistocene forest refugia. Mol Ecol 2009, 18:2668-2686.

19. Frati F, Hartl GB, Lovari S, Belibes M, Markov G: Quaternary radiation and genetic structure of the red fox (Vulpes vulpes) in the Mediterranean Basin, as revealed by allozymes and mitochondrial DNA. J Zool 1998, 245:43-51

20. Avise J: Molecular Markers, Natural History and Evolution New York: Chapman and Hall; 1994

21. Avise JC: Phylogeography: The history and formation of species Cambridge, Massachusetts and London: Harvard University Press; 2000.

22. Yang DY, Eng B, Waye JS, Dudar JC, Saunders SR: Improved DNA extraction from ancient bones using silica-based spin columns. Am J Phys Anthropol 1998, 105:539-543

23. Gilbert MTP, Bandelt HJ, Hofreiter M, Barnes I: Assessing ancient DNA studies. TREE 2005, 20:541-544.

24. Rozen S, Skaletsky HJ: Primer3 on the WWW for general users and for biologist programmers. In Bioinformatics Methods and Protocols: Methods in Molecular Biology. Edited by: Krawetz S. New Jersey: Humana Press; 2000:365-386

25. Posada D: jModelTest: Phylogenetic Model Averaging. Mol Biol Evol 2008, 25:1253-1256.

26. Guindon S, Gascuel O: A simple, fast, and accurate algorithm to estimate large phylogenies by maximum likelihood. Syst Biol 2003, 52:696-704.

27. Huelsenbeck JP, Ronquist F: MRBAYES: Bayesian inference of phylogeny. Bioinformatics 2001, 17:754-755.

28. Tamura K, Peterson D, Peterson N, Stecher G, Nei M, Kumar S: MEGA5: Molecular evolutionary genetics analysis using maximum likelihood, evolutionary distance, and maximum parsimony methods. Mol Biol Evol 2011, online early.

29. Clement M, Posada D, Crandall K: TCS: a computer program to estimate gene genealogies. Mol Ecol 2000, 9:1657-1660

30. Schneider S, Roessli D, Excoffier L: Arlequin: A software for population genetics data analysis. Genetics and Biometry Lab, Dept. of Anthropology.

31. Villesen P: FaBox: an online toolbox for fasta sequences. Mol Ecol Notes 2007, 7:965-968

32. Teacher AGF, Griffiths DJ: HapStar: automated haplotype network layout and visualization. Mol Ecol Res 2010, 11:151-153.

33. Ersts PJ: Geographic Distance Matrix Generator (version 1.2.3). [http:// biodiversityinformatics.amnh.org/open_source/gdmg].
34. Raymond M, Rousset F: GENEPOP (version 1.2): population genetics software for exact tests and ecumenicism. J Hered 1995, 86:248-249.

35. Rousset F: Genepop'007: a complete reimplementation of the Genepop software for Windows and Linux. Mol Ecol Res 2008, 8:103-106.

36. Drummond AJ, Rambaut A: BEAST: Bayesian evolutionary analysis by sampling trees. BMC Evol Biol 1007, 7:214-221

37. Firth C, Kitchen A, Shapiro A, Suchard MA, Holmes EC, Rambaut A: Using Time-Structured Data to Estimate Evolutionary Rates of Double-Stranded DNA Viruses. Mol Biol Evol 2010, 27:2038-2051.

38. Valdiosera CE, García N, Anderung C, Dalen L, Crégut-Bonnoure E, Kahlke RD, Stiller M, Brandström M, Thomas MG, Arsuaga JL, Götherström A, Barnes I: Staying out in the cold: glacial refugia and mitochondrial DNA phylogeography in ancient European brown bears. Mol Ecol 2007, 16:5140-5148.

39. Dalén L, Nystrom V, Valdiosera C, Germonpré M, Sablin M, Turner E, Angerbjörn A, Arsuaga JL, Götherström A: Ancient DNA reveals lack of postglacial habitat tracking in the arctic fox. PNAS 2007, 104:6726-6729.

40. Andersen KK, Svensson A, Johnsen SJ, Rasmussen SO, Biglera M, Röthlisberger R, Ruth U, Siggaard-Andersen ML, Steffensen JP, DahlJensen D, Vinther BM, Clausen HB: The Greenland Ice Core Chronology 2005, 15-42 ka. Part 1: constructing the time scale. Quaternary Sci Rev 2006, 25:3246-3257.

41. Gachot-Neveu H, Lefevre P, Roeder JJ, Henry C, Pulle ML: Genetic detection of sex-biased and age-biased dispersal in a population of wild carnivore, the red fox, Vulpes vulpes. Zool Sci 2009, 26:145-152.

42. Faunmap (1994): FAUNMAP: A database documenting late quaternaty distributions of mammal species in the United States Illinois: Illinnois State Museum, Scientific Papers; 1999

43. Harington C: Pleistocene mammals of the Yukon territory. PhD thesis University of Alberta; 1977

44. Soulsbury CD, Baker PJ, lossa G, Harris S: Fitness costs of dispersal in red foxes (Vulpes vulpes). Behavl Ecol Sociobiol 2008, 62:1289-1298.

45. Allen SH, Sargeant AB: Dispersal patterns of red foxes relative to population density. J Wildlife Manage 1993, 57:526-533.

46. Lehman N, Eisenhawer A, Hansen K, Mech DL, Peterson RO, Gogan PJ, Wayne RK: Introgression of coyote mitochondrial DNA into sympatric North American gray wold populations. Evolution 1991, 45:104-119.

47. Lehman N, Wayne RK: Analysis of coyote mitochondiral DNA genomtype frequencies: Estimation of the effective number of alleles. Genetics 1991, 128:404-416.

48. IUCN: The IUCN Red List of Threatened Species 2009.

49. Sommer RS, Benecke N: Late-Pleistocene and early Holocene hisory of the canid fauna of Europe (Canidae). Mamm Biol 2005, 70:227-241.

50. Hedges REM, Pettitt PB, Bronk Ramsey C, van Klinken GJ: ORAU Datalist. Archaeometry 1996, 38:391-415.

51. Street M, Baales M: Pleistocene/Holocene changes in the Rhineland fauna in a northwest European context. Archäologie in Eurasien 1999, 6:9-42.

52. Hock C: Das Magdalenien der Kniegrotte. Ein Hohlenfundplatz bei Dobritz, Saale-Orla Kreis. Weimarer Monografien zur Ur- und Frühgeschichte 2000, 35:1-202

53. Sommer RS, Nadachowski A: Glacial refugia of mammals in Europe: evidence from fossil records. Mammal Rev 2006, 36:251-265.

doi:10.1186/1471-2148-11-214

Cite this article as: Teacher et al: Modern and ancient red fox (Vulpes vulpes) in Europe show an unusual lack of geographical and temporal structuring, and differing responses within the carnivores to historical climatic change. BMC Evolutionary Biology 2011 11:214. 